Cahiers $d u$ MONDE RUSSE

\section{Cahiers du monde russe}

Russie - Empire russe - Union soviétique et États indépendants

$52 / 4 \mid 2011$

Varia

\title{
Anthony Heywood, Engineer of Revolutionary Russia
}

Juan Camilo Vergara

\section{OpenEdition \\ Journals}

Édition électronique

URL : http://journals.openedition.org/monderusse/7603

DOI : 10.4000/monderusse.7603

ISSN : $1777-5388$

\section{Éditeur}

Éditions de l'EHESS

\section{Édition imprimée}

Date de publication : 20 décembre 2011

Pagination : 719-721

ISBN : 978-2-7132-2353-2

ISSN : $1252-6576$

Référence électronique

Juan Camilo Vergara, "Anthony Heywood, Engineer of Revolutionary Russia », Cahiers du monde russe [En ligne], 52/4 | 2011, mis en ligne le 29 novembre 2012, Consulté le 22 septembre 2020. URL : http:// journals.openedition.org/monderusse/7603; DOI : https://doi.org/10.4000/monderusse.7603

Ce document a été généré automatiquement le 22 septembre 2020

(c) École des hautes études en sciences sociales 


\title{
Anthony Heywood, Engineer of Revolutionary Russia
}

\author{
Juan Camilo Vergara
}

\section{RÉFÉRENCE}

Anthony HEYWOOD, Engineer of Revolutionary Russia. Iurii V. Lomonosov

(1876-1952) and the Railways. Burlington : Ashgate Publishing, 2011, $x x x+400 p$.

1 Anthony Heywood nous offre dans son livre une vision innovante du «parcours ferroviaire » de la Russie de la fin du régime tsariste à l'installation des bolcheviks au pouvoir. Il s'inscrit dans une tendance biographique de l'histoire des sciences et des techniques, où l'individu jouit d'un intérêt particulier en tant que conducteur et développeur d'un savoir, partagé par certains à différents moments de l'histoire. Ce livre marque un tournant dans les publications de l'auteur qui, après s'être intéressé au rôle politique et économique des chemins de fer dans les révolutions de 1905 et 1917², illustre ses recherches par l'expérience d'un individu. C'est dans le cadre de ce récit qu'il retrace une vie morcelée en périodes, celle de l'ingénieur Jurij Lomonosov (1876-1952), dans laquelle nous apercevons le contexte de la Russie des années 1880 à 1935. Il nous offre une approche pertinente des continuités et des discontinuités apportées par les révolutions russes dans le monde ferroviaire et politique. Surtout, à travers la vie de cet ingénieur nous observons le rôle moteur joué par les chemins de fer dans les initiatives modernisatrices de l'État tsariste et de l'État soviétique.

2 S'intéressant aux chemins de fer comme révélateurs du fonctionnement étatique de ces périodes, A. Heywood parvient à combiner un solide corpus de sources primaires, parmi lesquelles nous soulignons la part des mémoires de Jurij Lomonosov. En outre, il fait appel à un ensemble important d'archives russes, auparavant inaccessibles aux chercheurs occidentaux, tout comme au journal intime et aux documents privés et professionnels de l'ingénieur qui ont survécu à ses constants déplacements. C'est justement grâce à ce croisement critique de sources qu'A. Heywood réussit à élaborer 
une analyse fine des complexités du personnage, reconstituant la vie d'un homme, d'un ingénieur et d'un haut responsable de l'administration tsariste et soviétique; une continuité pour le moins curieuse.

3 Il part ainsi à la recherche d'un acteur fortement engagé dans les événements politiques de son temps, à travers lequel nous apercevons des problématiques historiographiques propres à chaque période concernée. Sans tomber dans le piège de la succession de faits biographiques, A. Heywood s'engage, au contraire, dans une analyse critique sur un homme de son temps et sur le temps d'un homme. Il insiste sur cette vie riche en expériences, parfois dramatiques, toujours liée à l'industrialisation accélérée de la Russie sous Sergej Witte, jusqu'à la Première Guerre mondiale et à la révolution d'Octobre 1917. Il insiste particulièrement sur son rôle actif dans l'ensemble des systèmes politiques que connaît la Russie du début du siècle, non seulement en raison de ses aptitudes d'ingénieur, mais surtout par suite de ses convictions personnelles. Son expérience suit le prototype du spécialiste ferroviaire de la fin de l'époque tsariste et de la Russie soviétique des années 1920, avec son ascension professionnelle fulgurante, sa disgrâce progressive dans les années 1920 et finalement son exil au Royaume-Uni en 1927, période dans laquelle il est « effacé » de la mémoire soviétique.

4 Faisant partie de cette minorité d'historiens intéressés par le rôle des chemins de fer dans la Russie du début du $\mathrm{xx}^{\mathrm{e}}$ siècle, A. Heywood a raison de souligner que les scientifiques et les ingénieurs sont peu représentés par l'historiographie de l'époque révolutionnaire, une historiographie qui s'est souvent intéressée à des personnages appartenant, avant tout, au monde du politique (Stalin, Lenin, Trockij, etc.) ou des arts. Cet ouvrage est par conséquent une biographie critique de Lomonosov, un essai d'interprétation d'une époque à travers l'enfance, l'éducation et la vie professionnelle de cet homme, tout comme de ses sensibilités politiques et de sa tumultueuse vie privée.

5 Dès l'introduction, A. Heywood invite le lecteur à la réflexion afin de déterminer dans quelle mesure Lomonosov était le personnage que ses contradicteurs ont décrit; un homme atteint par des accusations de corruption et de détournement de fonds, aux prétendues sensibilités politiques radicales, mais signalé comme un antisoviétique opportuniste. À quel point sa biographie est-elle exemplaire du modèle de l'ingénieur russe du début de $\mathrm{xx}^{\mathrm{e}}$ siècle?

6 Malgré les problèmes d'interprétation que pose le traitement de certaines sources (notamment en ce qui concerne ses Mémoires, dans les premiers chapitres), le livre se structure de manière à éclairer systématiquement trois aspects de la vie du personnage : l'aspect professionnel, politique et privé. Dans ce but, l'auteur part de la petite enfance et de l'adolescence de Lomonosov (chapitre 1) pour comprendre comment les politiques d'élargissement de la base estudiantine, dans l'Institut des voies de communication, sont à l'origine de sa décision d'entrer dans le milieu ferroviaire. C'est dans le cadre des réformes de $\mathrm{S}$. Witte, en faveur de l'ingénierie civile durant les années 1890, que se décide la formation de Lomonosov au détriment d'une carrière militaire. Son expérience met en évidence le déclin et l'adaptation de la petite noblesse rurale aux nouvelles réalités économiques du pays.

7 Peu après, lorsque Lomonosov réussit son entrée dans la vie professionnelle, l'auteur décrit ses aptitudes pour la recherche et sa ténacité au sein d'un État qui, préoccupé avant tout par le contrôle des chemins de fer, n'accorde que peu de ressources à la 
recherche ferroviaire (chapitre 2). C'est pourquoi A. Heywood insiste sur l'importance qu'il y a à se demander si les instituts polytechniques ont su répondre aux impératifs d'industrialisation de la Russie et si le régime était lui-même capable de se réformer et de s'adapter aux exigences de son temps (chapitre 3). On voit apparaitre un Lomonosov plutôt libéral, frustré par un système auquel il n'a pas su s'accommoder, mais aux convictions ambiguës, qui participe activement aux événements révolutionnaires de 1905 à Kiev (chapitre 4). En effet, son prosélytisme révèle comment, dans les réunions politiques auxquelles il participe, l'écart entre libéraux et radicaux s'était opéré avant même la proclamation du manifeste d'octobre du tsar, traditionnellement vu comme la cause principale de la rupture entre ces deux camps.

8 Après une intéressante description de l'engagement de Lomonosov dans la fabrication d'engins explosifs, A. Heywood nous livre une image plus précise du personnage et de sa carrière au début du $\mathrm{xx}^{\mathrm{e}}$ siècle $:$ il s'agit du chapitre 5 , dans lequel l'auteur élargit ses sources. Le Lomonosov d'avant 1914 apparaît comme un cas singulier, il est l'exemple d'une réussite spectaculaire au sein du ministère des Voies de communication qui pousse l'auteur à relativiser les difficultés systémiques du régime tsariste à réformer son industrie ferroviaire. Lomonosov devient un haut responsable de l'administration impériale qui occupe des postes de recherche importants, si bien qu'il est l'un des pivots de la modernisation russe durant l'année 1917 et, bien au-delà, sous le gouvernement de Lenin (chapitre 8).

9 Se plaçant dans la perspective de la stratégie ferroviaire du régime bolchevik, A. Heywood retrouve Lomonosov au cœur de la récupération économique du pays dans les années 1920-1925 (chapitre 9). L'ingénieur, qui avait occupé de hautes responsabilités durant la Première Guerre mondiale, devient un intermédiaire avec l'étranger sans même être inscrit au parti bolchevik. L'auteur introduit à ce moment son journal intime et une série de sources soviétiques qui lui permettent de constater que le régime des années 1920-1925 avait comme priorité le développement des échanges économiques avec l'étranger. A. Heywood va même jusqu'à proposer l'hypothèse selon laquelle Lenin pensait nommer Lomonosov commissaire du peuple aux Voies de communication (p. 202), ce qui reste cependant à démontrer.

10 L'auteur poursuit avec une nécessaire et incontournable explication technique sur la révolution des locomotives à diesel tout au long du premier $\mathrm{xx}^{\mathrm{e}}$ siècle, dans laquelle Lomonosov joue un rôle important (chapitre 9). Cette participation le conduit au sommet de sa carrière au niveau international, au moment où il voit se dessiner sa disgrâce en URSS en raison de ses affaires de corruption et de son train de vie luxueux en Allemagne, qui culmine dans son exil. Au terme de sa carrière au service de l'État soviétique en 1927, il réussit, malgré tout, à garder son passeport soviétique qu'il renouvelle périodiquement jusqu'en 1945. L'un des rares cas où les autorités soviétiques ont autorisé l'un de leurs citoyens à vivre indépendamment à l'étranger. Quelles en furent les raisons? A. Heywood nous laisse malheureusement avec cette question.

11 Ce livre remarquable devrait constituer un encouragement à poursuivre des travaux historiographiques sur les chemins de fer russes. Mais son intérêt est aussi plus large : à travers la vie de Lomonosov, nous pouvons découvrir des caractéristiques communes aux régimes tsariste et soviétique. La biographie de ce personnage le place à mi-chemin de la rupture dans sa vie privée, en 1917, et les continuités de sa vie professionnelle durant les années 1920. 


\section{NOTES}

1. Notamment dans son livre Modernizing Lenin's Russia: Economic Reconstruction, Foreign Trade and the Railways, Cambridge : CUP, 1999. 